\title{
OSSOS. XIII. Fossilized Resonant Dropouts Tentatively Confirm Neptune's Migration Was Grainy and Slow
}

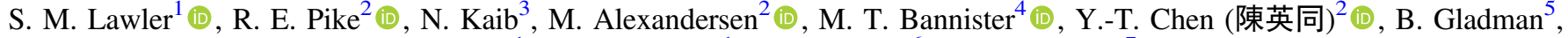 \\ S. Gwyn ${ }^{1}$, J. J. Kavelaars ${ }^{1}$ (1) , J.-M. Petit ${ }^{6}$ (i), and K. Volk ${ }^{7}$ (i) \\ ${ }^{1}$ Herzberg Astronomy and Astrophysics Research Centre, National Research Council of Canada, 5071 West Saanich Rd., Victoria, BC V9E 2E7, Canada \\ ${ }^{2}$ Institute of Astronomy and Astrophysics, Academia Sinica; 11F of AS/NTU Astronomy-Mathematics Building, No. 1 Roosevelt Rd., Section 4, Taipei 10617, \\ Taiwan \\ ${ }^{3}$ HL Dodge Department of Physics \& Astronomy, University of Oklahoma, Norman, OK 73019, USA \\ ${ }^{4}$ Astrophysics Research Centre, School of Mathematics and Physics, Queen's University Belfast, Belfast BT7 1NN, UK \\ ${ }^{5}$ Department of Physics and Astronomy, University of British Columbia, Vancouver, BC V6T 1Z1, Canada \\ ${ }^{6}$ Institut UTINAM UMR6213, CNRS, Univ. Bourgogne Franche-Comté, OSU Theta F-25000 Besançon, France \\ Lunar and Planetary Laboratory, University of Arizona, 1629 E. University Blvd., Tucson, AZ 85721, USA \\ Received 2018 July 27; revised 2019 April 5; accepted 2019 April 22; published 2019 June 4
}

\begin{abstract}
The migration of Neptune's resonances through the proto-Kuiper Belt has been imprinted in the distribution of small bodies in the outer solar system. Here we analyze five published Neptune migration models in detail, focusing on the high pericenter distance (high- $q$ ) trans-Neptunian objects (TNOs) near Neptune's 5:2 and 3:1 mean-motion resonances because they have large resonant populations, are outside the main classical belt, and are relatively isolated from other strong resonances. We compare the observationally biased output from these dynamical models with the detected TNOs from the Outer Solar System Origins Survey (OSSOS) via its Survey Simulator. All four of the new OSSOS detections of high- $q$ nonresonant TNOs are on the sunward side of the 5:2 and 3:1 resonances. We show that even after accounting for observation biases, this asymmetric distribution cannot be drawn from a uniform distribution of TNOs at $2 \sigma$ confidence. As shown by previous work, our analysis here tentatively confirms that the dynamical model that uses grainy slow Neptune migration provides the best match to the real high- $q$ TNO orbital data. However, due to extreme observational biases, we have very few high- $q$ TNO discoveries with which to statistically constrain the models. Thus, this analysis provides a framework for future comparison between the output from detailed, dynamically classified Neptune migration simulations and the TNO discoveries from future well-characterized surveys. We show that a deeper survey (to a limiting $r$-magnitude of 26.0) with a similar survey area to OSSOS could statistically distinguish between these five Neptune migration models.
\end{abstract}

Key words: celestial mechanics - Kuiper Belt: general

\section{Introduction}

The Kuiper Belt as observed today looks very different from the dynamically cold, flat disk of leftover planetesimals originally hypothesized to exist beyond Neptune's orbit (Edgeworth 1949). Several dynamically distinct components have been detected in the Kuiper Belt, some of which are highly excited, i.e., on very eccentric, inclined orbits (e.g., Gladman et al. 2008). The largest fraction of trans-Neptunian objects (TNOs) does indeed reside within the main classical belt on dynamically cold orbits. The Kuiper Belt also contains a large fraction $(\sim 20 \%)$ of TNOs on orbits that are in meanmotion resonances with Neptune (Gladman et al. 2012; Adams et al. 2014) and a significant scattering component that is on highly excited, dynamically unstable orbits (Gladman 2005; Shankman et al. 2013). Of interest in this work are the population of nonresonant TNOs with pericenters outside the gravitational scattering influence of Neptune: the "detached" population (Gladman et al. 2002).

Increasingly detailed dynamical simulations over the years have shown that much of the observed orbital structure of the Kuiper Belt can be caused by the outward migration of Neptune's orbit, with incrementally powerful constraints on the exact timing and mode of Neptune's migration (e.g., Malhotra 1993; Thommes et al. 1999; Tsiganis et al. 2005; Brasser \& Morbidelli 2013; Nesvorný \& Vokrouhlický 2016).
In order to compare these detailed simulations to observations of the distribution of TNO orbits, it is vital that observational biases are understood and accounted for. Particularly for the high pericenter distance (high- $q$ ) TNOs, the observational biases are severe and can have nonintuitive consequences for the detected orbital distributions (for a detailed discussion of these observational bias effects, see Shankman et al. 2017a). A thorough and well-tested way to account for observing biases is to carefully record a survey's depth, pointing direction and area on the sky, tracking fraction, and detection efficiency. These biases can then be applied to the output from a detailed dynamical simulation by Survey Simulator software, and the simulated detections can then be directly compared with the real survey detections in a statistically significant way, effectively "debiasing" the survey (Jones et al. 2006). This technique can only be applied to TNOs discovered in surveys that carefully record their biases; TNOs pulled from the Minor Planet Center (MPC) database without regard for discovery survey cannot be debiased because most surveys do not publish their biases and survey characteristics.

In this paper, we take the results from five recently published, detailed Neptune migration simulations and compare them to the detected TNOs from the Outer Solar System Origins Survey (OSSOS; Bannister et al. 2016, 2018), making use of the OSSOS Survey Simulator (Lawler et al. 2018a; Petit et al. 2018). We particularly focus on the high-pericenter TNOs 
as a powerful "fossilized" tracer of Neptune's migration. In Section 2 we discuss the dynamics of high- $q$ TNOs, as well as review in detail the properties of the migration simulations that we analyze (Section 2.1) and the structure of the near-resonant orbital element distributions for the different models (Section 2.2). In Section 3 we use the OSSOS Survey Simulator (Section 3.1) to apply the survey biases to the models, starting with a simple uniform distribution (Section 3.2), then observationally biasing the dynamical models (Section 3.3) and comparing them with the real OSSOS detections. While so few real detections provide little statistical constraint on the models, we conclude that overall, grainy slow (GS) Neptune migration provides the best match (Section 3.4), and this analysis provides a framework for how to compare these future TNO detections with the dynamical model output. We conclude with a comparison to the MPC database (Section 4.1) and a discussion of how resonant sticking may be important to explain high- $q$ TNOs on larger semimajor orbits (Section 4.2). In the Appendix, we provide detailed properties and analysis of a future, deeper survey that would be able to statistically distinguish between these migration models.

\section{High-pericenter TNOs as a Diagnostic of Neptune's Migration}

The TNOs with pericenters outside the gravitational scattering influence of Neptune either formed there (as did the classical Kuiper Belt on fairly circular orbits) or must have been emplaced later by some other interaction. Those that are not resonant and are on orbits that remain stable for $>10 \mathrm{Myr}$ timescales are part of either the classical belt or the "detached" TNO population, as they are currently dynamically decoupled from Neptune's scattering influence, unlike the "scattering" TNOs and Centaurs, which experience dynamical instability on $<10 \mathrm{Myr}$ timescales (Gladman et al. 2008). The border between the "scattering" and "detached" TNOs does not correspond to a simple cut in pericenter distance $q$, but $q>37$ au is often used to classify which TNOs are not currently experiencing strong gravitational encounters with Neptune (e.g., Lykawka \& Mukai 2007). For our purposes, we define both the outer classicals and the detached TNOs as part of the distant dynamically detached population. The outer classical TNOs are defined in Gladman et al. (2008) as $>10 \mathrm{Myr}$ stable orbits having $e<0.24$ and $a>47.8 \mathrm{au}$, the location of the 2:1 mean-motion resonance with Neptune, and the detached TNOs are defined in Gladman et al. (2008) as $>10 \mathrm{Myr}$ stable orbits having $e>0.24$ and $a>47.8$ au. Figure 1 shows the orbital properties of all of the detached and outer classical TNOs that were detected by the OSSOS ensemble of surveys (see Section 3.1).

Without a mechanism to create high- $q$ detached TNOs, there was originally no expectation of discovering eccentric high- $q$ TNOs. The first detections showed that a surprisingly large high- $q$ population was required due to the extreme observational biases against detecting these high- $q$ TNOs: their large pericenter distances mean that they always remain at large heliocentric distances and thus faint magnitudes (Gladman et al. 2002).

Gomes (2003) provided a possible explanation for these high- $q$ orbits, showing that Kozai cycling inside mean-motion resonances can simultaneously raise inclinations and pericenters. These TNOs are then more likely to fall out of resonance, because the resonances are thinner at the extreme values of

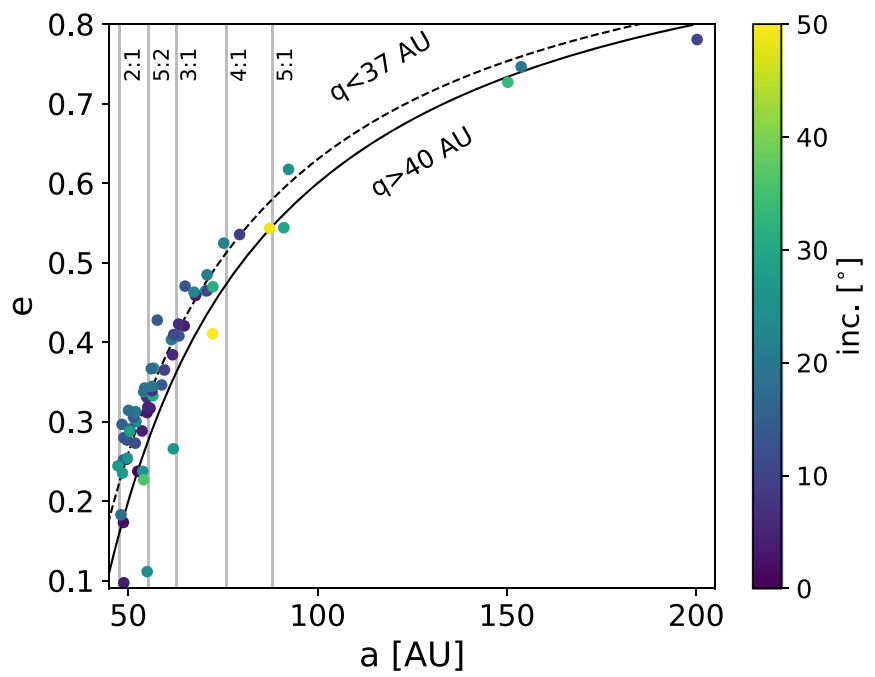

Figure 1. Orbital elements of the outer classical and detached TNOs detected by the OSSOS ensemble of surveys (Bannister et al. 2018); these are the nonresonant TNOs at larger $a$ than the 2:1 resonance that have stable orbits for at least $10 \mathrm{Myr}$. Inclination is shown by color. The solid curve shows $q=40 \mathrm{au}$, and the dashed curve shows $q=37 \mathrm{au}$. Points below the solid curve are high-pericenter TNOs $(q>40 \mathrm{au})$. The semimajor axes of several well-populated resonances are shown with gray lines for reference; all detected high- $q$ TNOs in the OSSOS ensemble are within \pm 2 au of strong resonances, which is the focus of this work.

inclination and eccentricity. The objects that fall out of resonance at the high- $e$ and low- $i$ end of a Kozai cycle are likely to be Neptune-crossing, and once outside the protection of a mean-motion resonance, they become part of the scattering population and are dynamically unstable on timescales of a few Myr. But the objects that fall out of resonance at the low- $e$ and high- $i$ end of a Kozai cycle are dynamically decoupled from Neptune due to their high pericenter distance $q$ and thus should remain in those orbits as a "fossilized" remnant of Neptune's past orbital migration history. Due to their long-term stability, these high- $q$ resonant dropouts are the focus of this work.

\subsection{Dynamical Simulations of Neptune's Migration}

Neptune's migration was first invoked to explain Pluto's eccentric resonant orbit (Malhotra 1993). Since those first Neptune migration models, many migration models and subsequent variations have been proposed, resulting in gradually better fits to the orbital structure of the steadily increasing number of known TNOs. The five models that we analyze here were chosen because they contain many particles and are dynamically classified according to the criteria laid out in Gladman et al. (2008; as is the case for Pike \& Lawler 2017), ${ }^{8}$ or because the authors provided their simulation output in a manner that was able to be dynamically classified through additional dynamical simulations (as is the case for models from Kaib \& Sheppard 2016). ${ }^{9}$

Pike et al. (2017) and Pike \& Lawler (2017, hereafter PL17) presented a detailed analysis of the giant-planet migration simulation from Brasser \& Morbidelli (2013). The planetary orbital evolution path within this simulation is based on the "Nice model" simulation from Levison et al. (2008). The Nice

\footnotetext{
8 The dynamically classified model from Pike et al. (2017) is publicly available at doi:10.11570/16.0009.

9 The dynamically classified model from Kaib \& Sheppard (2016) is publicly available at doi:10.11570/19.0008.
} 
model (Thommes et al. 1999; Tsiganis et al. 2005) invokes dynamical instability and scattering between the giant planets, causing Neptune to attain high eccentricity (maximum $e \simeq 0.3$ ) as it migrates outward while damping to a more circular orbit by scattering planetesimals. Neptune's high-eccentricity phase in this model has been demonstrated to cause problems with the structure of the classical belt (Dawson \& Murray-Clay 2012), the resonant TNO populations (Gladman et al. 2012), and the asteroid belt (Roig \& Nesvorný 2015). Subsequent simulations show that limiting Neptune's maximum eccentricity and migration timescale can preserve the cold classical population (Batygin et al. 2011; Dawson \& Murray-Clay 2012; Ribeiro de Sousa et al. 2018), but it is difficult to create this starting condition for Neptune. However, the detailed structure is testable because of the large number of particles in this simulation, and if other aspects of the outer solar system structure provide a compelling match to the real detections, then it would be worthwhile to consider ways to include a high$e$ phase of Neptune in migration models. It is also a simpler model in some ways than models that require an additional giant planet that is later ejected. This simulation produces "beards" of objects that drop out of resonance on both sides of the resonance, caused by the combination of Kozai cycling and narrowing of the resonance as Neptune's orbit circularizes.

Kaib \& Sheppard (2016, hereafter KS16) provided a detailed model of the outer Kuiper Belt structure resulting from grainy or smooth migration combined with slow or fast migration timescales. These four KS16 models build upon previously published work and agree with similar simulations in Nesvorný et al. (2016). In each of the KS16 simulations, Neptune is scattered but never attains a very high eccentricity $(e<0.1)$, and the migrations all include a "jump" in $a$ and $e$ meant to simulate the scattering of a now-ejected ice giant (as in Nesvorný 2015b), sometimes referred to as the "jumping Jupiter" model (Brasser et al. 2009; Morbidelli et al. 2010). Two of the models include only smooth migration (Malhotra 1993; Hahn \& Malhotra 2005), and two use "grainy migration," first discussed in Nesvorný \& Vokrouhlický (2016) and further analyzed in Nesvorný et al. (2016). In grainy migration, Neptune has small jumps in semimajor axis to simulate scattering of the largest planetesimals (roughly $\sim 1000 \mathrm{~km}$, or Pluto-sized), and this affects capture and retention of TNOs in resonances and appears to produce an overall Kuiper Belt structure that more closely matches observations (Nesvorný \& Vokrouhlický 2016). More recent work (Shannon \& Dawson 2018) uses binaries, cold classical TNOs, and resonant TNOs to place a limit on the number of $\sim 1000 \mathrm{~km}$ planetesimals that could have existed prior to Neptune's migration, which agrees with the previous analysis. The timescales for these migrations have $e$-folding timescales of $10 \mathrm{Myr}$ pre-jump and $30 \mathrm{Myr}$ post-jump for the fast migrations and $30 \mathrm{Myr}$ pre-jump and $100 \mathrm{Myr}$ post-jump for the slow migrations. These timescales are shown to provide good matches to the inclination distribution of TNOs (Nesvorný 2015a).

To avoid confusion, we define TNOs closer to the Sun than a given resonance as "sunward" and those more distant as "outward." Both Nesvorný et al. (2016) and KS16 show "trails" of particles that drop out of the resonances on the sunward side as the resonances march outward with Neptune's migration. It was shown by KS16 that this effect is stronger in grainy migration than smooth migration and stronger in slower migration than faster migration.
Throughout the paper, we use the following abbreviations to refer to the five simulations.

1. All KS16 simulations keep Neptune at relatively low eccentricity $(e<0.1)$ with a jump in Neptune's $a$ and $e$ that is caused by scattering of a now-ejected additional ice giant.

(a) KS16 GF: grainy migration with a fast timescale (efolding timescale of 10 and $30 \mathrm{Myr}$ pre- and postNeptune's large jump in $a$, respectively).

(b) KS16 GS: grainy migration with a slow timescale ( $e$ folding timescale of 30 and $100 \mathrm{Myr}$ pre- and postNeptune's large jump in $a$, respectively).

(c) KS16 SmF: smooth migration with the same fast timescale above.

(d) KS16 SmS: smooth migration with the same slow timescale above.

2. PL17: Neptune scatters to high eccentricity $(e \simeq 0.3)$ and circularizes while migrating outward with a timescale of 100 Myr.

The general effect of Kozai cycling combined with Neptune's migration is different for each of the five simulations we analyze. Most of the dropout appears to happen during Neptune's circularization phase in PL17, resulting in rather symmetric "beards" of near-resonant objects at high- $q$ (see Figure 1 in that work). The four simulations in KS16 form "trails" of near-resonant, high- $q$ objects preferentially populating the sunward side of the resonances (see Figure 2 in that work). The trails are of varying properties and densities, depending on the simulation. Grainy migration appears to more easily drop objects out of resonance, as does slower-timescale Neptune migration.

\subsection{The 5:2 and 3:1 Resonances}

We focus on the 5:2 and 3:1 resonances in this analysis because they are powerful resonances, relatively isolated in semimajor axis, less contaminated by resonant sticking (unlike more sunward resonances that are embedded in the classical Kuiper Belt), and close enough that surveys have a significant number of detected TNOs both inside the resonance and at nearby semimajor axes. The OSSOS ensemble of surveys includes TNO detections around each of these resonances, so it is possible to make a statistical comparison of the real detections to the simulation results. This requires a careful selection of real TNOs and a dynamical classification of the orbits of model objects to diagnose resonance and nonresonance (see Section 3.1).

The particles within the five simulations have been classified into dynamical classes using 10-30 Myr orbital integrations including the four giant planets to diagnose resonance occupation (following the TNO classification scheme in Gladman et al. 2008). The PL17 simulation particles are dynamically classified in Pike et al. (2017), while the KS16 particles are classified here. The orbital structure around the 5:2 and 3:1 resonances is shown for all five simulations in Figure 2 (5:2 in left panels, 3:1 in right panels). In each of the plots, circles show particles that are resonant at the end of the simulation, with point size proportional to libration amplitude, and nonresonant particles are shown with crosses. Color shows inclination, ranging from purple showing the lowest inclinations to yellow showing the highest inclinations $\left(\sim 50^{\circ}\right.$; same 
Near the 5:2 Resonance

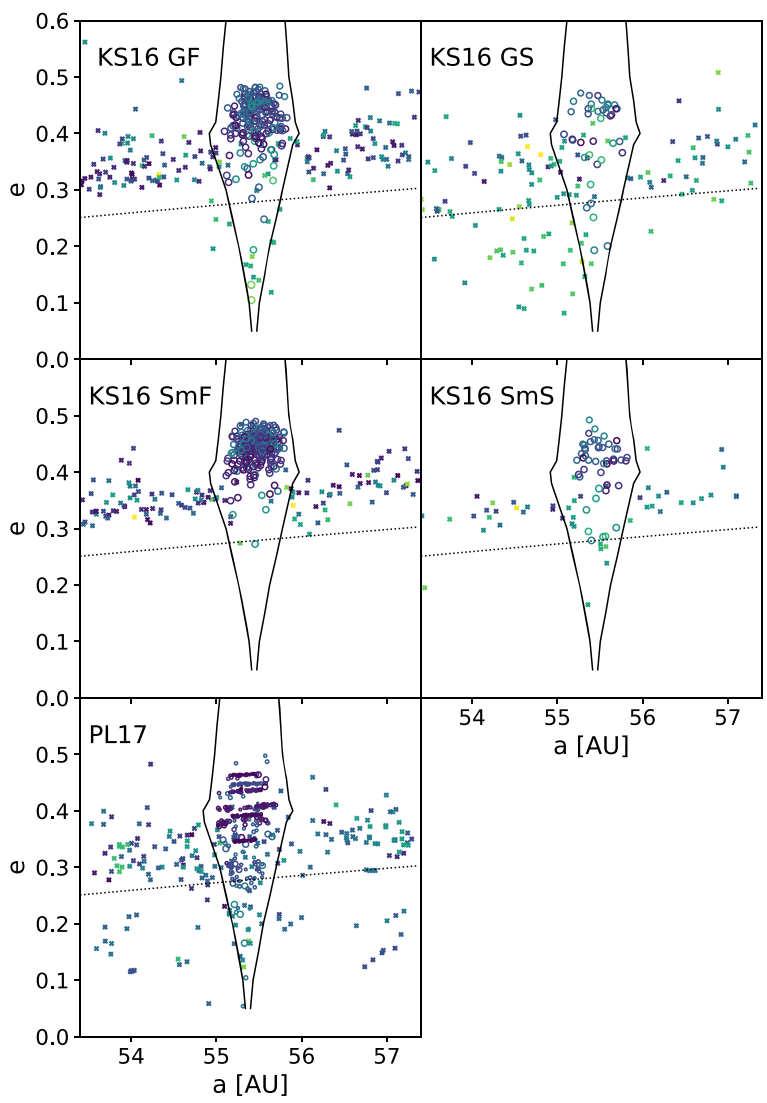

Near the 3:1 Resonance

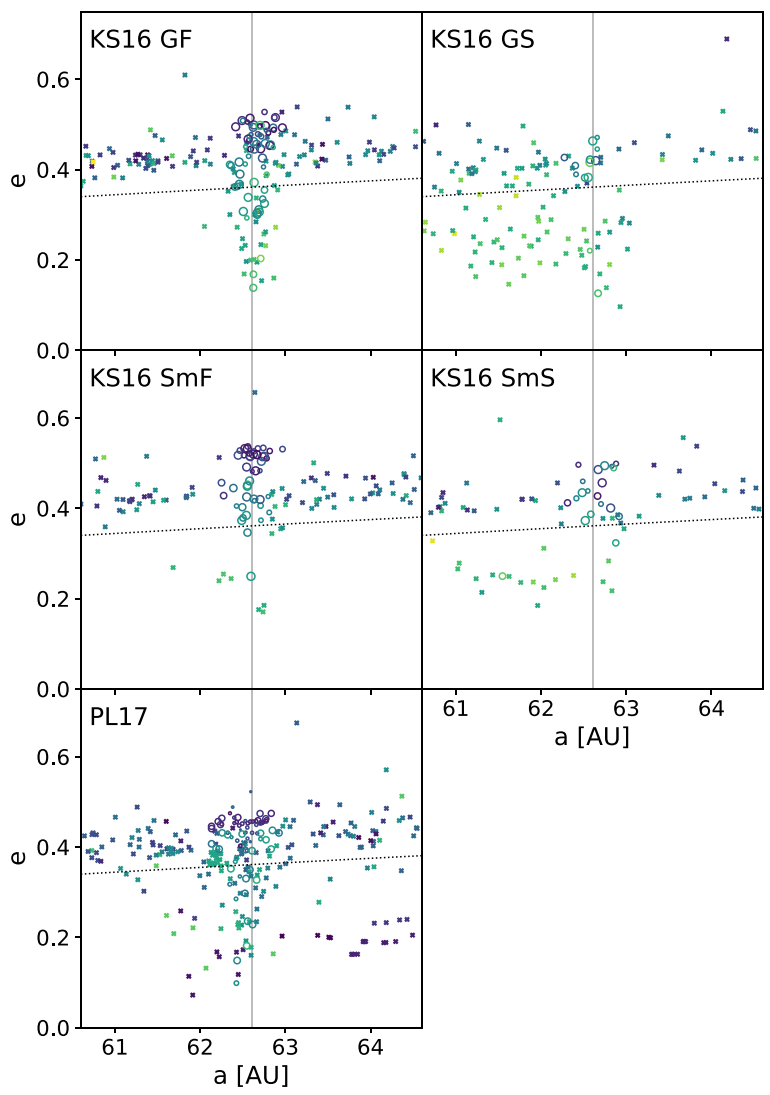

Figure 2. Comparison between the simulated objects with $a$ within \pm 2 au of the 5:2 ( $a=55.4$ au; left panels) and 3:1 ( $a=62.6$ au; right panels) resonance centers. Circles show objects that we classify as resonant with circle size proportional to libration amplitude, crosses show nonresonant objects, and color shows inclination (same color scale as in Figure 1). The objects located below the dotted black curve have $q>40$ au and are the high-pericenter objects we use for comparison (Section 3). The gray solid line (right panels) shows the 3:1 resonance center, while the solid black lines (left panels) show the 5:2 resonant stability limits computed in Malhotra et al. (2018) as a guide; our dynamical classification generally agrees with these limits.

color scale as in Figure 1). The points below the black dotted curve are objects with $q>40$ au.

Some general trends are worth discussing in the five simulations. The resonant dropouts (high- $q$ objects marked by crosses) are almost entirely sunward of the resonances in the two grainy KS16 simulations, nearly absent in the two smooth KS16 simulations for the 5:2 but present sunward for the 3:1, and present on both the sunward and outward sides of the resonances in the PL17 simulation. The high- $q$ resonant dropouts in the grainy KS16 simulations are, on average, of higher inclination that the lower- $q$ (higher- $e$ ) particles in the simulation (as shown by yellow and green rather than blue and purple). This supports the idea that the Kozai effect is important in these resonant dropouts: high- $q$ (and correspondingly low-e) particles have high inclinations when they fall out of resonance and enter the fossilized detached population. The highinclination effect is not as obvious in the Nice model PL17 particles. This is likely due to Neptune's initially high eccentricity ( $e \simeq 0.3$ ) causing very wide resonances in $a$. The combination of Neptune's orbital circularization causing the resonance boundaries to narrow and Kozai oscillations caused many objects to drop out of resonance on both the sunward and outward sides.
There are several higher-order resonances close to the 5:2 resonance that each have TNOs observed to be currently occupying these resonances (e.g., the OSSOS ensemble discovered TNOs occupying the 7:3,12:5, 13:5, and 8:3 resonances, all with $a$ within \pm 2 au of the center of the 5:2 resonance; Bannister et al. 2018). There are no known TNOs within \pm 2 au of the 3:1 resonance that occupy a resonance other than the 3:1. All of the known resonant TNOs within \pm 2 au of the $5: 2$ in resonances other than the $5: 2$ have $q<40$ au and thus do not fall into our high- $q$ sample. For the high- $q$ particles created in the course of the Neptune migration simulations, we check that the pericenter raising occurs primarily inside the 5:2 and not inside the nearby resonances by checking resonance occupation at the time step where a particle first attains $q>40$ au (shown in Figure 3). The number of particles with period ratios close to the 5:2 when attaining $q>40$ au exceeds the number close to the other resonances (particularly the 12:5, which is closest) by a factor of several.

The next step is to compare these models with the real detected TNOs. However, a direct comparison between the models and the detected TNOs would be misleading because of the severe detection biases based on distance and survey characteristics. We are able to compare the carefully selected 


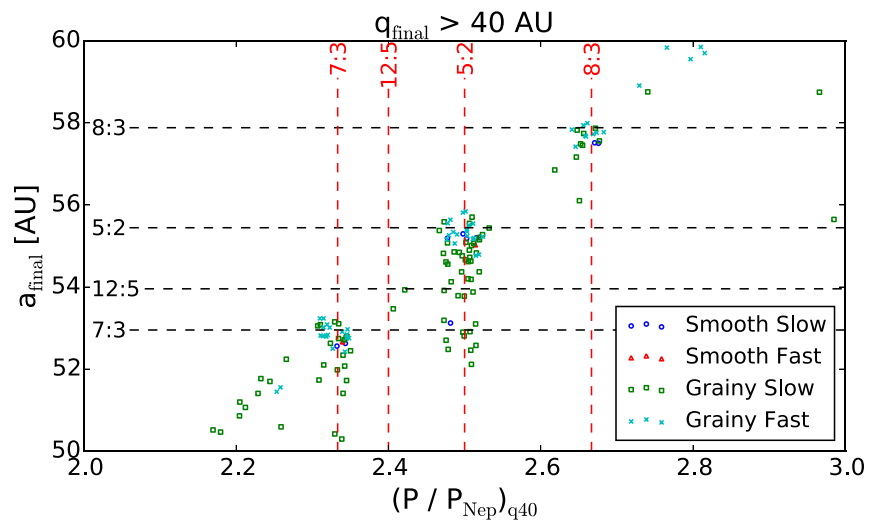

Figure 3. Every particle that ever attains high pericenter $(q>40 \mathrm{au})$ in the KS16 models and their instantaneous period ratio with Neptune at the time step where the particle first attained $q>40$ au ( $x$-axis), compared with the object's final $a$ (y-axis). The high- $q$ objects in all of the KS16 simulations that end the simulation with $a$ close to the 5:2 (those near the black horizontal dashed 5:2 line) first attain their high- $q$ almost exclusively inside the 5:2 (that is, they cluster along the red vertical dashed $5: 2$ line), so contamination by pericenter raising inside other nearby resonances is not a significant effect.

near-resonant test particles with the real TNOs near resonance by using the OSSOS Survey Simulator.

\section{Accounting for Observational Biases: Comparing Models to Observations}

\subsection{The Well-characterized Outer Solar System Origins Survey}

The OSSOS (Bannister et al. 2016, 2018) was a large program on the Canada-France-Hawaii Telescope over 5 yr. It is a wellcharacterized survey, where all of the survey block pointings, detection efficiencies, and tracking efficiencies and rate cuts at different magnitude limits are well known and published, so all of these biases can be applied to a dynamical model output, and the forward-biased model can be directly statistically compared to the detected TNOs in the survey (Lawler et al. 2018a). OSSOS, in combination with three other well-characterized surveys (Petit et al. 2011, 2017; Alexandersen et al. 2016) is referred to as the "OSSOS ensemble" of surveys and, in total, detected 1142 TNOs with exceptionally well-measured orbits. This Survey Simulator ${ }^{10}$ methodology has previously been used to constrain the size distribution and population of scattering TNOs (Shankman et al. 2013, 2016; Lawler et al. 2018b), the populations and orbital structure within different mean-motion resonances (Gladman et al. 2012; Lawler \& Gladman 2013; Pike et al. 2015; Alexandersen et al. 2016; Volk et al. 2016, 2018), and the populations and size distributions of the main classical belt (Petit et al. 2011). The Survey Simulator methodology has been used in several previous papers to statistically compare dynamical simulations with observed TNO distributions. These works have provided support for grainy migration (Nesvorný \& Vokrouhlický 2016), made predictions about observations to test the Nice model (Pike et al. 2017), and placed constraints on the population, orbital distribution, and mass of the distant Kuiper Belt in the presence of an undiscovered giant planet (Lawler et al. 2017, 2018a; Shankman et al. 2017b).

This survey characterization is critical for quantifying selection effects that might affect the interpretation of OSSOS detections. The biases against detection of high- $q$ TNOs are extreme and

\footnotetext{
${ }^{10}$ The OSSOS Survey Simulator software is publicly available at https:// github.com/OSSOS/SurveySimulator.
}
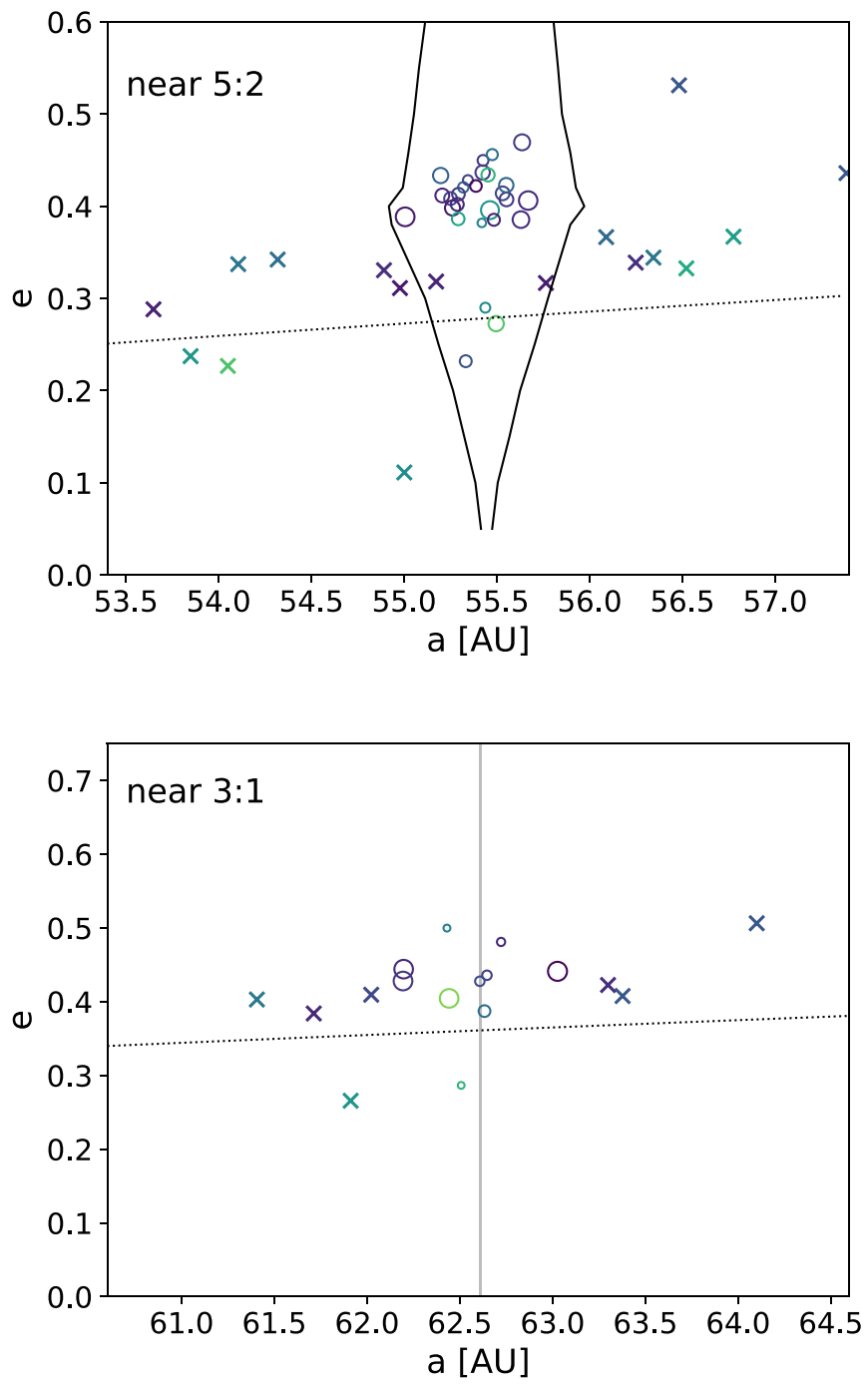

Figure 4. All of the TNOs detected by OSSOS within \pm 2 au of the 5:2 and 3:1 resonances (top and bottom panels, respectively). The TNOs that are not in the $5: 2$ or $3: 1$ resonances are indicated by crosses, while circles show 5:2 and 3:1 resonators, with larger circle sizes showing larger libration amplitudes. Color indicates inclination, as in Figure 1.

unintuitive (Shankman et al. 2017a), so using a Survey Simulator to test and account for these biases is vital. The near-resonant TNOs do not have a longitudinal bias, but the sunward TNOs are slightly more detectable than the outward TNOs because of their smaller semimajor axes. The Survey Simulator and characterized survey are crucial to understanding the significance of this effect and the relative detectability of nearby resonant TNOs and to interpreting the detected sunward/outward fractions.

We focus on the OSSOS TNO detections within \pm 2 au of the 5:2 and 3:1 resonances, shown in Figure 4. The TNOs in this $a$ range that are not in the $5: 2$ or $3: 1$ resonances are indicated by a cross, while TNOs in the 5:2 and 3:1 resonances are shown by circles, with larger circles indicating larger libration amplitudes within the resonance.

\subsection{Sunward versus outward Near-resonant High-q Objects: Not a Uniform Distribution}

The OSSOS discovered a number of near-resonant and high$q$ objects. We focus on the 5:2 and 3:1 resonances because these resonances are relatively isolated and, as demonstrated in 
Table 1

High- $q$ Near-resonant TNOs Detected by the OSSOS Ensemble

\begin{tabular}{|c|c|c|c|c|c|c|c|c|c|}
\hline $\begin{array}{l}\text { OSSOS } \\
\text { Name }\end{array}$ & $\begin{array}{c}\text { MPC } \\
\text { Designation }\end{array}$ & $\begin{array}{c}a \\
(\mathrm{au})\end{array}$ & $e$ & $\begin{array}{c}i \\
(\mathrm{deg})\end{array}$ & $\begin{array}{c}q \\
(\mathrm{au})\end{array}$ & $\begin{array}{c}\Omega \\
(\mathrm{deg})\end{array}$ & $\begin{array}{c}\omega \\
(\mathrm{deg})\end{array}$ & $\begin{array}{c}\text { T peri } \\
\text { (JD-2,400,000) }\end{array}$ & $\begin{array}{c}H_{g} \\
(\mathrm{mag})\end{array}$ \\
\hline \multicolumn{2}{|c|}{ 5:2 resonance: } & $55.4^{\mathrm{a}}$ & & & & & & & \\
\hline L5c16 & $2005 \mathrm{CG}_{81}$ & 53.849 & 0.23735 & 26.153 & 41.068 & 134.664 & 56.94 & 67,197 & $6.14^{\mathrm{b}}$ \\
\hline o5m86 & $2015 \mathrm{KQ}_{174}$ & 55.00 & 0.111 & 24.398 & 48.89 & 213.97 & 290.20 & 25,373 & 7.27 \\
\hline \multicolumn{2}{|c|}{ 3:1 resonance: } & $62.6^{\mathrm{a}}$ & & & & & & & \\
\hline
\end{tabular}

Notes. For errors on measured orbital elements, see Bannister et al. (2018). All digits given are significant.

${ }^{\mathrm{a}}$ For reference, we give $a$ for the 5:2 and 3:1 resonances from $a_{\mathrm{N}}(p / q)^{2 / 3}$.

${ }^{\mathrm{b}}$ Here $H_{r}$ has been converted to $H_{g}$ using the relation $g-r=0.7$; see Shankman et al. (2016).

Section 2.2, are responsible for the vast majority of the high- $q$ TNOs fossilized within \pm 2 au of those resonances. The OSSOS ensemble (Bannister et al. 2018) detected 27 TNOs currently librating in the 5:2 resonance and 11 in the 3:1 resonance. Within \pm 2 au of the 5:2 resonance, OSSOS detected three nonresonant TNOs with $q>40$ au, all sunward of the resonance: $\mathrm{L} 5 \mathrm{cl} 6\left(2005 \mathrm{CG}_{81}\right)$, HL7j $5\left(2007 \mathrm{LE}_{38}\right)$, and $\circ 5 \mathrm{~m} 86\left(2015 \mathrm{KQ}_{174}\right)$. Within \pm 2 au of the $3: 1$ resonance, OSSOS detected one nonresonant TNO with $q>40$ au, also sunward of the resonance: $03172\left(2013 \mathrm{SK}_{100}\right)$. These high- $q$ TNOs are listed in Table 1. We test the significance of the discovery of all four near-resonant objects sunward of the resonances by using the OSSOS Survey Simulator to account for discovery biases.

Comparing the OSSOS detections to a uniform distribution around the resonances indicates that the intrinsic distribution is significantly nonuniform. We created orbital distributions of high- $q$ objects around the 5:2 and 3:1 MMR. We used the combined objects from all KS16 models with $q>40$ au and period ratios of 2.4-2.6 $P_{N}$ and 2.9-3.1 $P_{N}$ for the 5:2 and 3:1, respectively. Using the $q$ and $i$ distributions of these objects, we then randomly sampled from the synthetic objects assuming a uniform distribution in this range of period ratios. Orbital angles were randomly assigned, and the synthetic objects were input into the Survey Simulator. The near-5:2 simulations ran until three objects were detected, and the near-3:1 simulations ran until just one object was detected. The maximum $a$ from each run was recorded and compared to the maximum $a$ of the actual detections 1000 times, as shown in Figure 5. The maximum $a$ of the real high- $q$ nonresonant detections (55.0 au for the 5:2 and 61.9 au for the 3:1) are unlikely to be drawn from an underlying uniform distribution. We find that the maximum $a$ of the near-5:2 population is less than or equal to 55.0 au (the maximum $a$ of the real OSSOS catalog) only $5.9 \%$ of the time. Moreover, in the bottom panel, we see that the detection probability of near-3:1 objects is nearly uniform across the 3:1 resonance. If we account for the fact that the only real near-3:1 detection is on the sunward side of the resonance, we find a $2.9 \%$ probability that OSSOS would have detected such an asymmetric high- $q$ population if the underlying distribution was uniform within \pm 2 au of both resonances.

The OSSOS ensemble of surveys can discriminate between different distributions of near-resonant TNOs, which are known to depend on the specifics of planetary migration, as discussed in Section 2.1. Using the OSSOS Survey Simulator, we reject the simple uniform distribution at $2 \sigma$ significance. However, the predicted particle distributions resulting from migration are typically more complicated than this simple model. We test the
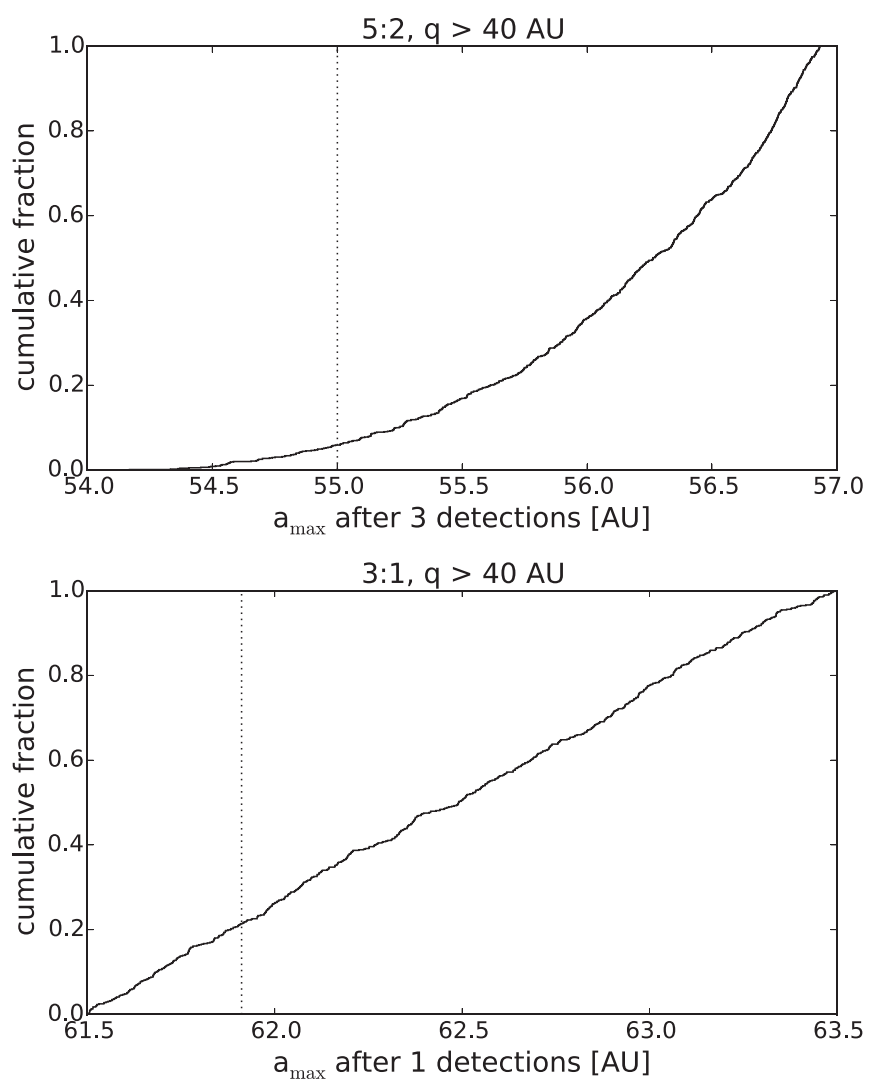

Figure 5. Largest- $a$ objects detected from a uniform period ratio distribution for three detections near the 5:2 resonance (top panel; resonance center at $a=55.4 \mathrm{au}$ ) and one detection near the 3:1 resonance (bottom panel; resonance center at $a=62.6 \mathrm{au}$ ). A distribution of high $q$ TNOs drawn from a uniform distribution of period ratios was supplied to the Survey Simulator, which was run until the same number of detections as the OSSOS ensemble surveys was found (three near-5:2 objects and one near-3:1 object). The largest $a$ of the real detections near each resonance is shown by the dashed line. Using the OSSOS Survey Simulator, we find that the likelihood that the underlying distribution is uniform is $5.9 \%$ for the $5: 2$ and $20 \%$ for the $3: 1$, so the likelihood of both distributions finding low- $a$ members is $2.9 \%$.

specific model particle distributions from different planetary migration simulations in the following section.

\subsection{Using the Survey Simulator to Test the Detailed Models}

The Survey Simulator software works by randomly drawing an orbit from a file or parametric distribution, choosing an absolute $H$ magnitude for that drawn object based on an $H$ distribution model, then determining if that drawn object was 
bright enough and in the right position on the sky to have been detected by the OSSOS ensemble of survey pointings (the Survey Simulator methodology is discussed extensively in previous works, e.g., Lawler et al. 2018a, and references therein). In order to make sure that we properly measure the population of high- $q$ resonant dropouts predicted by each model, we compare simulated detections from survey-biased models where there are the same number of simulated detections of resonant objects as real resonant TNO detections in OSSOS. Because of this scaling and the different detection biases for resonant TNOs, we must carefully preserve the resonant or nonresonant conditions of test particles while we randomize model orbits, another reason this analysis works best with a dynamically classified model.

To produce an orbital distribution from the output of a dynamical simulation, as we have done here, orbits are randomly drawn from the model end-state, with each line in the model orbital distribution file being equally likely to be chosen. If the drawn orbit is classified as nonresonant, the orbits will precess over time, so the angular orbital elements (the argument of pericenter $\omega$, longitude of the ascending node $\Omega$, and mean anomaly $\mathcal{M}$ ) are all chosen randomly to simulate the distribution over long timescales. Resonant orbits have a longitude bias: they come to pericenter preferentially at specific positions on the sky. Thus, the on-sky pointing positions of the survey are important and can cause nonintuitive detection biases for different resonances (see, e.g., Figure 1 in Gladman et al. 2012). To preserve this longitude bias, resonant orbits must be randomized in a specific way. As part of the classification into resonances (as discussed in detail in Pike et al. 2017), we record the libration amplitude $\Delta \phi_{j k}$ and libration center $\left\langle\phi_{j k}\right\rangle$ of each resonant test particle, which limits where the test particle can come to pericenter (and thus be most detectable) relative to Neptune's position. For the 5:2, all resonant particles have $\left\langle\phi_{52}\right\rangle=180^{\circ}$, but the $3: 1$ has symmetric librators with $\left\langle\phi_{31}\right\rangle=180^{\circ}$ and asymmetric librators with $\left\langle\phi_{31}\right\rangle \simeq 80^{\circ}$ or $280^{\circ}$. The libration amplitude distribution is different in every simulation, as is the relative population of the different 3:1 resonant islands, but we preserve this distribution inside the Survey Simulator. For each resonant orbit drawn from the file, following Gladman et al. (2012), the value of $\phi_{j k}$ is chosen sinusoidally according to the libration amplitude around the resonance center. The mean anomaly $\mathcal{M}$ and longitude of the ascending node $\Omega$ are then chosen randomly, and the argument of pericenter $\omega$ is chosen to satisfy the resonant condition: $\phi_{j k}=j \lambda_{\mathrm{TNO}}-k \lambda_{\mathrm{N}}-(j-k)$ $\left(\Omega_{\mathrm{TNO}}+\omega_{\mathrm{TNO}}\right)$, where $\lambda=\Omega+\omega+\mathcal{M}$ and the subscripts "N" and "TNO" refer to Neptune and the resonant test particle/ TNO, respectively. In this way, we can randomize orbits and draw many thousands of orbits from a slice of a model that may contain only a few hundred orbits and still be consistent with the unique resonant dynamics of each model's output. After the orbits are randomized, an $H$ magnitude is assigned from a literature size distribution (Lawler et al. 2018b) in the range $4.5<H_{r}<11$, which covers the range of OSSOS detections in the population we are testing. The instantaneous distance of the test particle is then used to assign an apparent magnitude, which, together with the test particle's instantaneous on-sky position and rate of motion, is used to determine whether or not this particular particle is detected within the survey characteristics.

We use the Survey Simulator in several different ways to compare the models with the real OSSOS detections near the
5:2 and 3:1 resonances and to assess how well each model matches reality.

Figure 6 is an example of the detailed analysis that can be done with dynamically classified models containing many test particles and Survey Simulator software combined with detections from a well-characterized survey. We divide the models into $a, e$ bins; assess the detectability of each bin using the OSSOS Survey Simulator; and compare the survey-biased results with the real detections. The Survey Simulator is run until 100 objects are detected in each $a, e$ bin (preserving resonance and nonresonance for each drawn model object as described above). Detectability is measured by counting how many simulated objects were drawn to reach 100 simulated detections. In the $a, e$ bins where detectability is low, more simulated objects must be drawn to reach 100 simulated detections than where detectability is high. The total detectability is then normalized by requiring the same total number of detections as in OSSOS near the 5:2 and 3:1 resonances. The simulated and real detections in each bin can then be directly compared as shown in Figure 6, where darker green shows $a, e$ bins where too few simulated detections are produced by the model, darker pink shows bins where too many simulated detections are produced by the model, lighter colors show good matches between the simulation and reality, and white shows bins with no simulated or real detections. (The color scale is normalized the same for all panels around both the 5:2 and 3:1, but the two resonances do not have the same normalization because of the different number of detections).

Some interesting features are immediately obvious in Figure 6. Every single model underpredicts the number of TNOs observed at relatively high-e inside the 5:2 and 3:1 resonances (green squares near the center of each panel). We speculate that this has something to do with the dynamical excitation of TNOs immediately prior to being captured, and this may be an important feature to attempt to reproduce in future dynamical simulations of Neptune migration. The numbers of detected TNOs are so small for the high- $q$ (low-e) TNOs that it is impossible to evaluate which model here provides the best match in this parameter space. We turn to another simulation method for a more direct test.

Table 2 shows the result of running each model through the Survey Simulator until it produces the same number of resonant detections as discovered by the OSSOS ensemble. In order to not be strongly affected by a single random draw, we repeated this 1000 times. Table 2 gives this average number of high- $q$ near-resonant detections produced by each model when the Survey Simulator produces the same number of resonant detections as in the real OSSOS ensemble. The comparison between the fraction of detected sunward/outward high- $q$ objects in each simulation is one way to measure the goodness of fit of these models to the data. We also provide the intrinsic populations of high- $q$ nonresonant objects for each model, again scaled so that there are the same number of resonant objects as discovered by OSSOS (obviously, the intrinsic population would have to be much larger than the detected population; the scaling is done to provide ease of comparison between models and also to show the relative over-/underpopulation of the sunward/outward populations). As discussed before, because of the complicated survey biases, we do not compare the intrinsic sunward/outward fractions directly to the real OSSOS detections but instead compare the observationally biased models. As another way of evaluating the models, we 

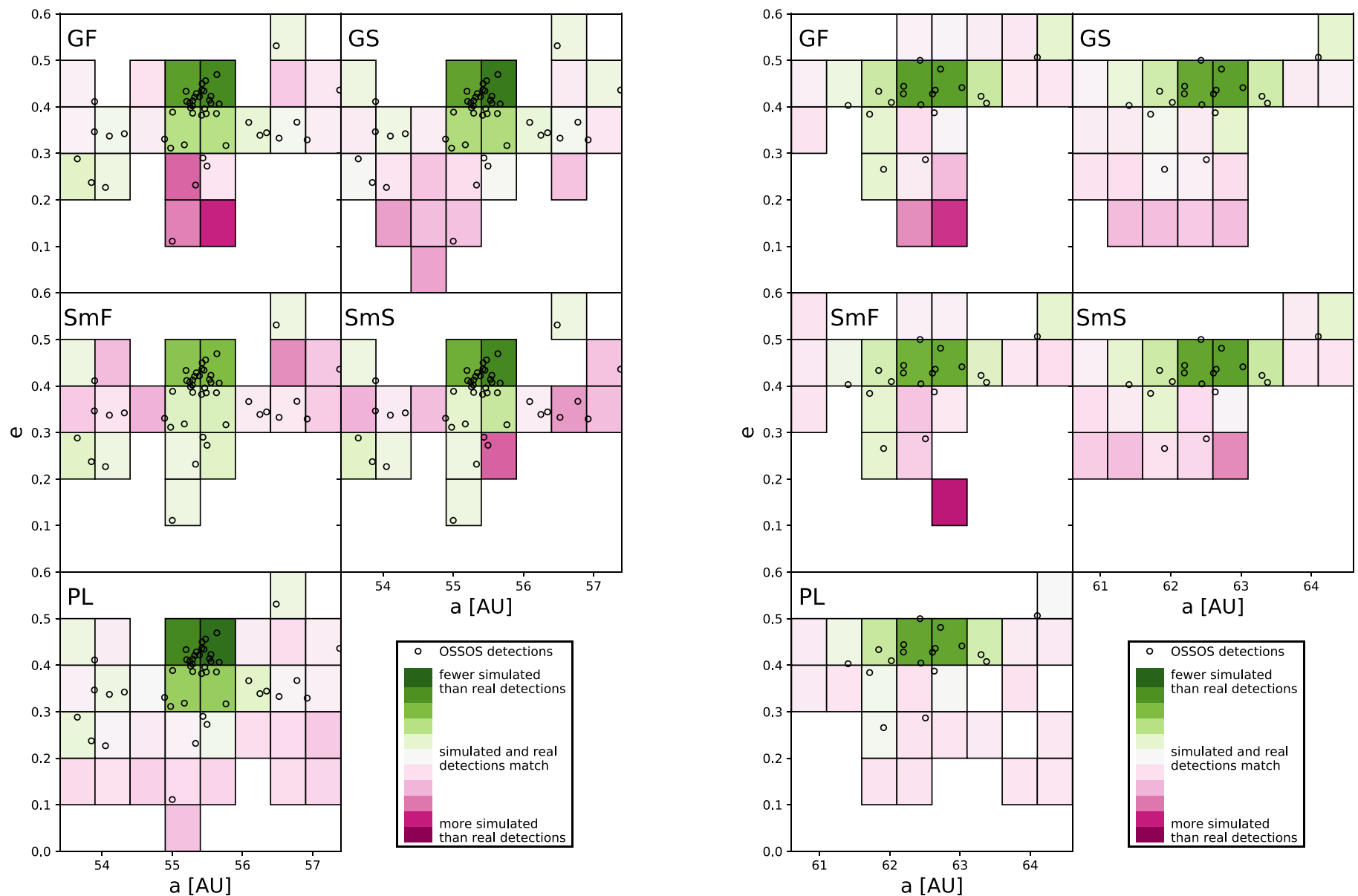

Figure 6. Detectability of objects in each dynamical model compared with the number of real OSSOS detections near the 5:2 (left panels) and 3:1 (right panels) averaged over many Survey Simulator runs. See text for the definition of detectability. Circles show the location of real OSSOS TNOs in $a, e$, and colored squares show the number of simulated detections from the models compared with real detections in each $a, e$ bin. Panels are on the same scale for each resonance, with darker pink showing where the model produces more simulated than real TNO detections, darker green showing more real TNOs than simulated TNO detections, and gray showing where the match is perfect.

measure the number of Survey Simulator runs where the exact OSSOS sunward/outward number of detections is matched exactly for each resonance.

Looking at the 5:2 portion of Table 2, it is clear that the KS16 GS simulation provides the best match to the OSSOS data. The average number of sunward and outward high- $q$ objects detected is the closest to the real OSSOS detections, and the fraction of runs where exactly three sunward and zero outward high- $q$ objects are detected is the highest. The PL17 model is the second-best match for the 5:2. Coincidentally, the PL17 intrinsic sunward/outward population ratios are actually closest to the OSSOS detections, but when biased by the Survey Simulator, they drop to much lower values than the real OSSOS detections, with only $1 \%$ of the Survey Simulator runs producing exactly three high- $q$ sunward and zero outward detections along with the 27 5:2 resonant detections. Interestingly, the KS16 grainy fast (GF), smooth fast (SmF), and smooth slow (SmS) produce zero or near-zero runs where there is an exact match to the OSSOS high- $q$ detections. This is because the resonant population is much higher relative to the nonresonant in these simulations than in the GS and PL17 models, so there are simply not enough nonresonant detections by the time the Survey Simulator has produced 27 5:2 resonant detections in each of these three models. For the near-3:1 objects, the KS16 GS simulation provides too many high- $q$ detections both sunward and outward, while the KS16 SmS migration produces the best fit, though we note that with only one high- $q$ OSSOS detection to compare here, this analysis holds little statistical weight and is mainly qualitative.

\subsection{GS Migration Likely Provides the Best Match}

There are many possible ways to test dynamical models against the OSSOS data; we remind the reader that here we are focusing only on the high- $q$ resonant dropouts because their "fossilized" dynamically detached state means that particles that manage to get into this parameter space will stay on a very similar orbit on Gyr timescales. Comparison of orbital elements, libration amplitude distributions, and Kozai fractions within the resonances has already been done for the PL17 simulation in that work. Comparison between the models and OSSOS resonant data will be in upcoming OSSOS team papers specifically focusing on the discoveries and orbital structure of the 5:2 and 3:1 resonances.

The number of high- $q$ nonresonant detections produced by the KS16 GS model when matching the OSSOS resonant detections agrees well for the 5:2 (Table 2). However, when comparing the near-3:1 simulated detections, the number predicted by the KS16 GS model is far too large compared 
Table 2

Resonant and Near-resonant Detected and Intrinsic Populations

\begin{tabular}{|c|c|c|c|c|c|c|}
\hline \multirow{3}{*}{ Source } & \multicolumn{3}{|c|}{ Normalized to 27 5:2 Resonant TNOs } & \multicolumn{3}{|c|}{ Normalized to 11 3:1 Resonant TNOs } \\
\hline & \multirow{2}{*}{$\begin{array}{c}\text { Intrinsic Populations } \\
\text { High- } q \text { Near-5:2 } \\
\text { Sunward/Outward }\end{array}$} & \multicolumn{2}{|c|}{ Biased by Survey ("Detections") } & \multirow{2}{*}{$\begin{array}{c}\text { Intrinsic Populations } \\
\text { High- } q \text { Near-3:1 } \\
\text { Sunward/Outward }^{\text {a }}\end{array}$} & \multicolumn{2}{|c|}{ Biased by Survey ("Detections") } \\
\hline & & $\begin{array}{c}\text { High- } q \text { Near-5:2 } \\
\text { Sunward/Outward }\end{array}$ & $\begin{array}{c}\text { Fraction of } \\
\text { Matching Runs }\end{array}$ & & $\begin{array}{c}\text { High- } q \text { Near-3:1 } \\
\text { Sunward/Outward }\end{array}$ & $\begin{array}{c}\text { Fraction of } \\
\text { Matching Runs }\end{array}$ \\
\hline \multicolumn{7}{|l|}{ Models } \\
\hline KS16 GF & $0.5 / 1.2$ & $0.1 / 0.1$ & 0.00 & $0.6 / 0.8$ & $0.1 / 0.2$ & 0.09 \\
\hline KS16 GS & $20.2 / 2.0$ & $3.1 / 0.3$ & 0.16 & $38.9 / 11.0$ & $9.3 / 2.4$ & 0.01 \\
\hline KS16 SmF & $0.1 / 0.0$ & $0.0 / 0.0$ & 0.00 & $1.0 / 1.5$ & $0.1 / 0.2$ & 0.08 \\
\hline KS16 SmS & $1.1 / 2.3$ & $0.1 / 0.4$ & 0.00 & $7.7 / 2.2$ & $1.0 / 0.3$ & 0.24 \\
\hline PL17 & $2.8 / 1.8$ & $0.4 / 0.2$ & 0.01 & $5.9 / 5.4$ & $1.4 / 1.5$ & 0.08 \\
\hline \multicolumn{7}{|l|}{ Real TNOs } \\
\hline OSSOS + & & $3 / 0$ & & & $1 / 0$ & \\
\hline
\end{tabular}

Notes.

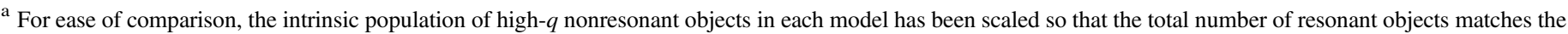
number of OSSOS detections (27 5:2 resonators and 11 3:1 resonators; the near-5:2 and near-3:1 populations are scaled independently from each other).

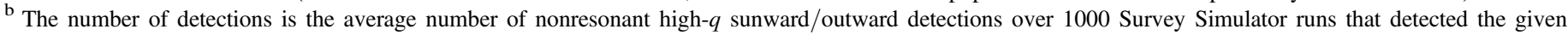
number of 5:2 (27) or 3:1 (11) resonators with different random number seeds (see Section 3.3).

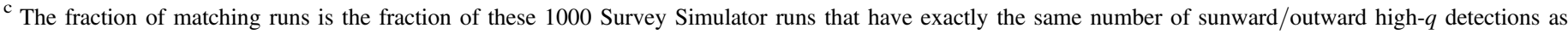
OSSOS for each resonance.
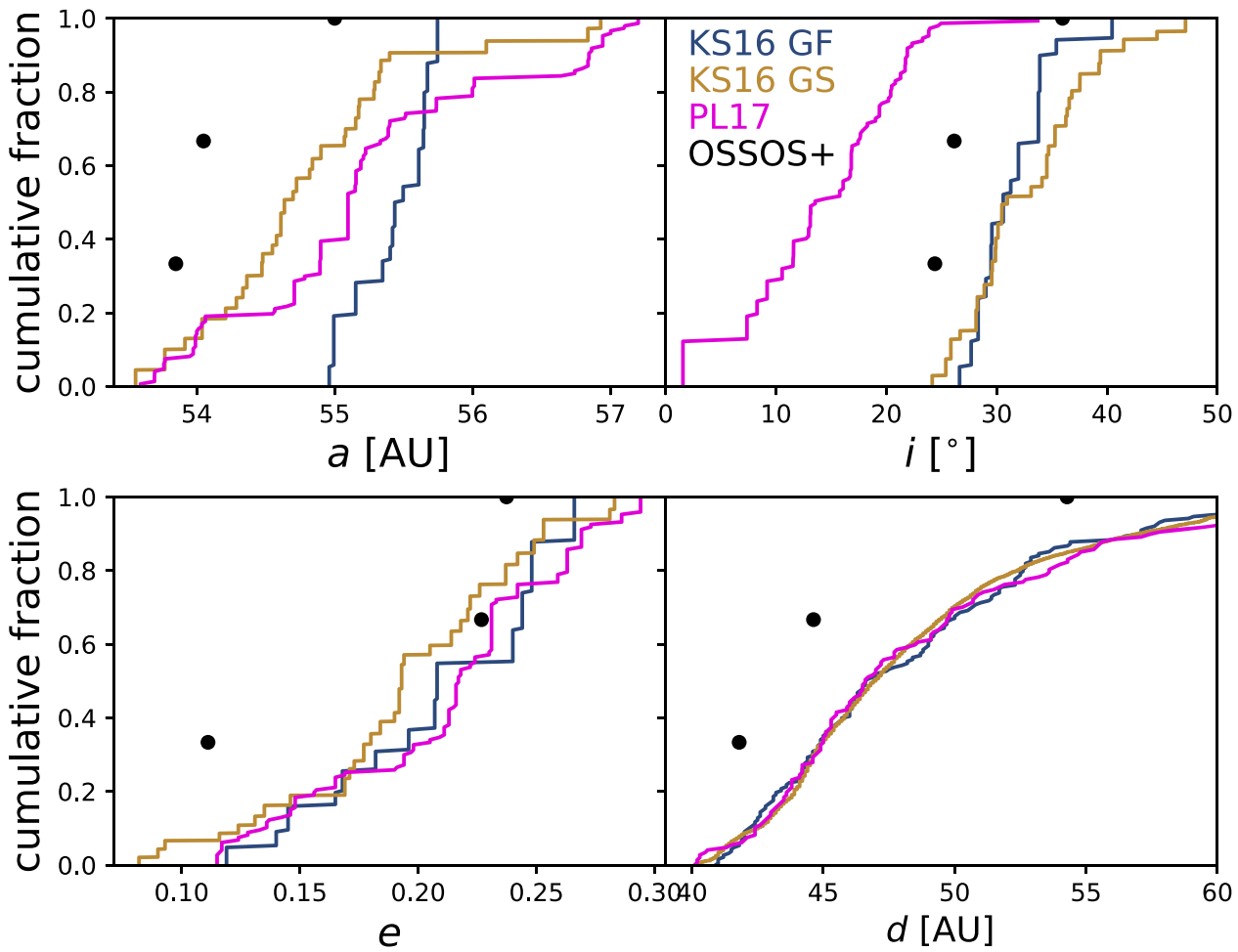

Figure 7. Comparison between the three high- $q$ OSSOS detections (black points) and the properties of simulated nonresonant high- $q$ detections from three of the models that have a significant number of high- $q$ nonresonant objects near the 5:2. Because of the very small number of real OSSOS detections in this parameter space, we present this mainly as a guide for future comparisons; see discussion in the Appendix. Only KS16 GF is statistically ruled out by this analysis; it does not have a wide enough range of $a$ or $i$ to encompass the real OSSOS detections.

with OSSOS, and the PL17 and KS16 SmS models provide a better match. But with only one high- $q$ detection near the $3: 1$, it is extremely difficult to say anything significant about these comparisons.

Another way to compare the simulated detections is provided in Figure 7, where the three high- $q$ near-5:2 OSSOS detections are compared with the cumulative distributions of the simulated model detections of high- $q$ objects for three of the models that have the most high- $q$ objects near the 5:2. The KS16 GS and PL17 models both easily provide more sunward than outward of the 5:2 resonance, while the KS16 GF provides detections only in a very narrow range of $a$. The PL17 model provides overall lower inclinations in the high- $q$ population than either the KS16 GS or GF models, but it is unclear from the small number of detections which provides a better match to the OSSOS data. Only the KS16 GF model can be ruled out: 

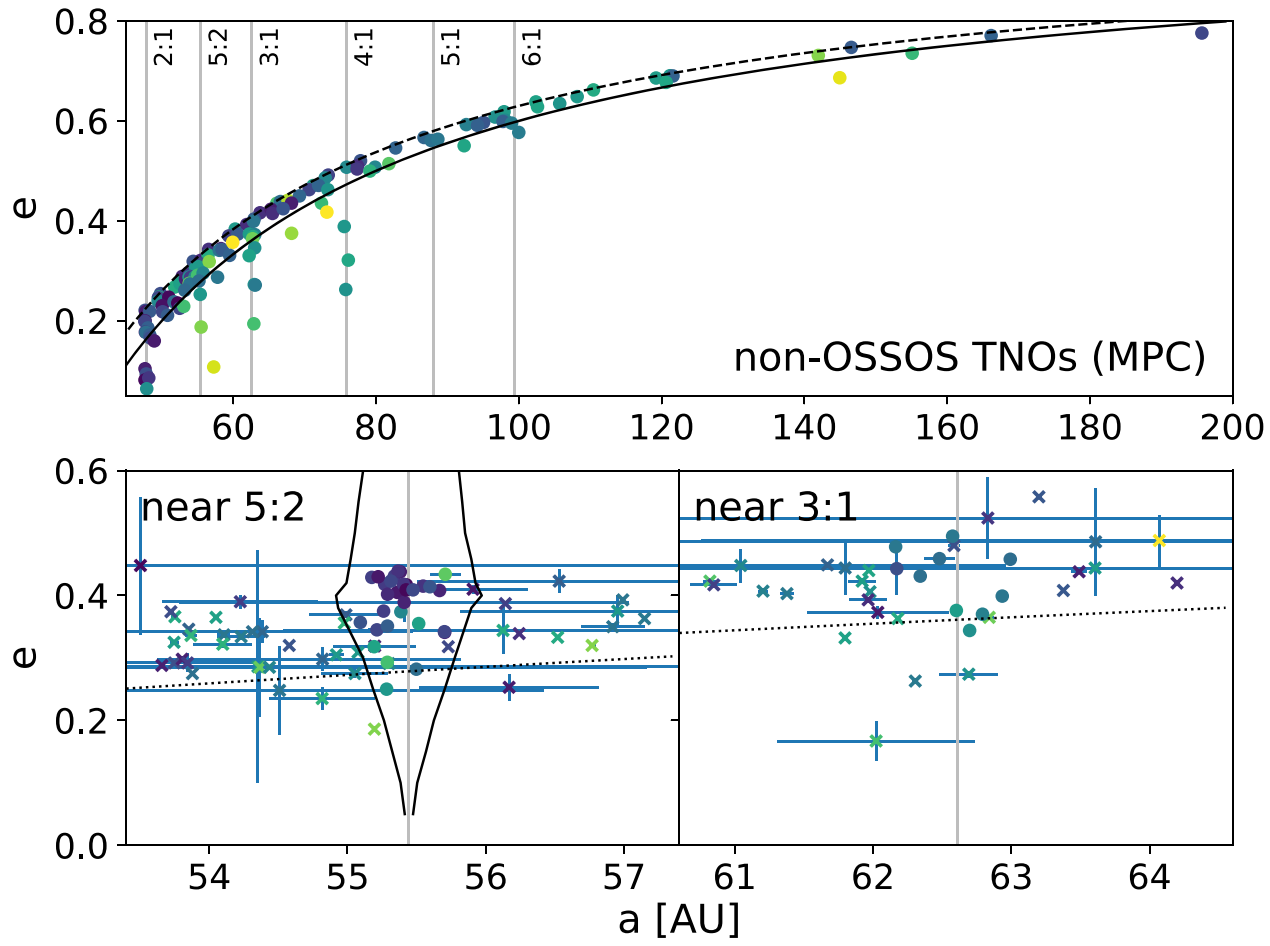

Figure 8. Orbital elements of TNOs from the MPC database as of 2018 December with $q>37$ au, $a>47.6$ au (outward from the location of the 2:1 resonance), and an MPC uncertainty parameter of $<6$, excluding those detected by the OSSOS ensemble. The color scale for inclination is the same as in Figure 1. In the top panel, the solid and dashed black lines show $q=40$ and 37 au, respectively. In the bottom panels, the dotted line shows $q=40$ au. The top panel can be compared to the real OSSOS detections in Figure 1, and the bottom two panels can be compared with the real OSSOS detections in Figure 4. In the bottom panels, orbital classification has been performed in the same manner as for OSSOS, and circles show TNOs that librate in the 5:2 and 3:1 resonances. Most of these TNOs have orbital uncertainties that are so large that they are not useful for the sort of analysis performed here; additionally, observation biases for these TNOs are unknown.

due to the fast migration timescale, resonant dropouts appear to only happen very close to the end position of the resonance, keeping a very narrow $a$ distribution that does not encompass the range of OSSOS detections. Using the Anderson-Darling (AD) test (Anderson \& Darling 1954), neither the PL17 nor KS16 GS models are formally rejectable by the data presented here. The eccentricity distribution between the three models is remarkably similar, as is the distribution of distances where objects are detected. We reiterate that with so few detections, Figure 7 does little more than provide guidelines for future work that will hopefully provide far more high- $q$ TNO detections (see the Appendix).

\section{Discussion}

\subsection{Comparison with the MPC Database}

Our analysis has focused only on TNOs detected in wellcharacterized surveys; here we also compare with all of the detected TNOs currently listed in the MPC database (as of 2018 December). There are more TNOs listed in the MPC database than the OSSOS ensemble, but they come from a myriad of surveys with unreported observation biases, so direct comparison between models and detections is impossible without a large uncertainty. Figure 8 shows all TNOs in the MPC database with $a$ larger than the 2:1 resonance center and $q>37$ au. Due to the very large uncertainties on most of the data, we did not perform dynamical classification to determine resonance occupation or scattering behavior.

The bottom panels of Figure 8 show the near-5:2 and near3:1 TNOs, which can be compared to the OSSOS detections plotted in Figure 4. The pointings of the OSSOS were specifically chosen to optimize the detection of $n: 2$ resonators (Bannister et al. 2016), so there are many more 5:2 resonant detections in OSSOS than MPC TNO detections inside the Malhotra et al. (2018) 5:2 stability limits. Other than a lack of dynamically excited 5:2 resonators (almost certainly an observation bias effect), the MPC database detections are completely consistent with the OSSOS detections. We do not include these extra TNOs in our analysis because the observation biases are completely unknown; thus, the detailed observational bias analysis performed above is not possible for this sample.

\subsection{The Dynamics of Resonant Dropouts}

The orbital distribution of these fossilized resonant dropouts is a window into the construction of our solar system's architecture. The interplay of Kozai oscillations within resonances and Neptune's migration appears to explain much of the resonant and nonresonant Kuiper Belt dynamical structure that we observe. Grainy migration gives us information not only on Neptune's migration but also on the number of large $(\sim 1000 \mathrm{~km})$ planetesimals that formed before Neptune's migration occurred. The analysis performed here provides additional support for the idea that there were hundreds of such large planetesimals present by the time of Neptune's migration, as per Shannon \& Dawson (2018), and supports the theory of Nesvorný \& Vokrouhlický (2016).

While GS migration appears to provide a satisfactory explanation for the high- $q$ TNOs at $a<100 \mathrm{au}$, it is not clear if this could also be applied to TNOs at much larger distances. Recent simulations by $\mathrm{Yu}$ et al. (2018) show that at any given moment, for TNOs with 30 au $<a<100$ au, there are of order 
the same number of scattering TNOs as transiently resonant TNOs. Transient sticks to mean-motion resonances by the unstable scattering population provide a nice explanation for the very large populations present in very distant mean-motion resonances (Gladman et al. 2012; Pike et al. 2015; Bannister et al. 2017; Holman et al. 2018; Volk et al. 2018). Pike et al. (2015) performed a detailed analysis of TNOs in and around the 5:1 resonance and showed that there is a nonresonant near$5: 1$ TNO in a high- $q$, long-term stable orbit sunward of the resonance. At $a=88$ au, the 5:1 is not expected to have any primordial TNO population and must be entirely populated by resonant sticking from the scattering population in any of the Neptune migration models we discuss in this work. Just after the Nice model planet-scattering event, the scattering population would have been much larger; the scattering population today is likely $<1 \%$ of its original population (Duncan \& Levison 1997). Resonant sticking by this much larger scattering population at early times, combined with grainy Neptune migration, could explain high- $q$ TNOs at much larger semimajor axes.

The two highest- $q$ TNOs discovered to date have pericenter distances more than 25 au larger than the next highest- $q$ TNOs, possibly implying a different formation mechanism than other high- $q$ TNOs: Sedna has $a=507$ au and $q=76 \mathrm{au}$, and $2012 \mathrm{VP}_{113}$ has $a=266 \mathrm{au}$ and $q=80 \mathrm{au}$. For the same resonant sticking, Kozai $q$ lifting, and resonant dropout narrative to work for these two extreme TNOs, they must have been caught into very large $a$ resonances. Sedna is near the semimajor axis that would correspond to its 69:1 resonance, and $2012 \mathrm{VP}_{113}$ is close to the 105:4 resonance. Initially, it seems a bit ridiculous to think that these extremely high-order, distant resonances could possibly affect TNOs, but resonance occupation by real TNOs has been shown in such high-order resonances as, e.g., the 27:4 and 35:8 at $a=108$ and 81 au, respectively (Bannister et al. 2018). Additionally, simulations by Gallardo et al. (2012) found resonance sticking with pericenter lifting out to the outermost semimajor axes they tested at $500 \mathrm{au}$, and test particles within these simulations had their pericenters raised up to $q \simeq 70$ au. However, they showed that for $q$ to change dramatically, inclinations generally must be high $\left(i \gtrsim 40^{\circ}\right)$ when particles are first captured into resonance, and these TNOs' inclinations do not appear to be high enough for this explanation to be compelling: Sedna has $i=11^{\circ}$, and $2012 \mathrm{VP}_{113}$ has $i=24^{\circ}$. There are many suggested explanations for distant, high- $q$ TNOs in the literature, but using Neptune's migration in combination with resonance sticking is an intriguing and much simpler possible explanation for all but the highest- $q$ TNOs and thus merits further exploration in detailed simulations. Repeating Neptune migration simulations with many more scattering test particles at large $a$ and carefully classifying and analyzing test particle behavior near the distant resonances is a highly fruitful route to measuring the effectiveness of this population mechanism and may provide an answer to the source of many of the fossilized high- $q$ objects.

\section{Summary and Conclusions}

The current observations of the outer solar system show that the distributions of high- $q$ particles near the 5:2 and 3:1 resonances are not uniform. The analysis here has shown that more high- $q$ TNOs on the sunward side of these two resonances is not an observational bias effect, and the structure is likely due to resonant dropouts during Neptune migration. The distribution of these near-resonant particles provides a powerful discriminating tool for assessing how well different models of planetary migration reproduce the outer solar system. The current four characterized near-resonant TNO detections, all on the sunward side of nearby resonances, provide a statistically significant rejection of a uniform distribution and indicate that GS Neptune migration (as in Kaib \& Sheppard 2016) provides the closest match to the observed near-resonant TNO distribution.

Out of the five Neptune migration simulations analyzed, the simulation that used grainy Neptune migration with slower timescales and a scattering jump that keeps Neptune at $e<0.1$ best reproduces the relative number and distribution of nearresonant high- $q$ TNOs observed by OSSOS, but there simply are not yet enough high- $q$ TNOs to provide statistically robust support for any of the models we tested above the others. We hope this paper has provided a framework to rigorously test future observational data sets against upcoming dynamical models.

The Large Synoptic Survey Telescope (LSST) is expected to detect hundreds of new TNOs because of its incredible sky coverage and cadence. The LSST main survey of 18,000 deg 2 has a single-image limiting magnitude of $m_{r}=24.5$ (Ivezić et al. 2019; Jones et al. 2016), shallower than much of OSSOS. It will thus provide comparatively few high- $q$ TNOs, due to the steep TNO size distribution. However, a deep drilling survey of only a few suitably sited pointings, with a comparatively sparse cadence, may also be able to differentiate between these models. The most powerful way to test migration models will be through a deeper targeted TNO survey, such as the proposed survey we modeled for the Subaru Hyper Suprime-Cam (HSC; see the Appendix), which would detect enough high- $q$ TNOs to distinguish between these five migration models at high statistical significance.

Additionally, larger-scale Neptune migration simulations with more scattering particles can test the effectiveness of resonant sticking by scattering TNOs during Neptune's migration phase for producing very high- $q$ TNOs at large semimajor axes, such as Sedna. Due to the extreme biases against observing these extremely high- $q$ TNOs and the low number of detections, the best way to understand their population is through dynamical modeling in combination with a Survey Simulator to fully account for these severe observation biases.

By reconstructing the details of Neptune's migration through detailed study of the Kuiper Belt's orbital structure, we learn about possible additional, now-ejected giant planets (as suggested by the "jumping Jupiter" model; Nesvorný 2015b) and the size and number of planetesimals that formed at this distance, since grainy migration requires a large number of relatively large $(\sim 1000 \mathrm{~km})$ planetesimals (Nesvorný \& Vokrouhlický 2016). The large OSSOS data set and Survey Simulator, which are both publicly available, provide the most powerful way to test these and future dynamical evolution models of the solar system. Using a Survey Simulator is the best way to take into account the complicated observational biases in the outer solar system, particularly in the high- $q$ and resonant TNO populations, and we hope that this will become the standard for testing the validity of dynamical models. 
The authors acknowledge the sacred nature of Maunakea and appreciate the opportunity to observe from this mountain. The CFHT is operated by the National Research Council (NRC) of Canada, the Institute National des Sciences de l'Universe of the Centre National de la Recherche Scientifique (CNRS) of France, and the University of Hawaii, with OSSOS receiving additional access due to contributions from the Institute of Astronomy and Astrophysics, Academia Sinica, Taiwan. Data are produced and hosted at the Canadian Astronomy Data Centre; processing and analysis were performed using computing and storage capacity provided by the Canadian Advanced Network For Astronomy Research (CANFAR). This research has made use of data and/or services provided by the International Astronomical Union's Minor Planet Center.

The authors thank R. Brasser for providing the output of his simulation for this analysis, C. Shankman for legacy code and helpful conversations, H. Ngo for useful conversations, and an anonymous referee for suggestions that improved the focus of the paper. S.M.L. gratefully acknowledges support from the NRCCanada Plaskett Fellowship. M.T.B. appreciates support from UK STFC grant ST/P0003094/1. K.V. acknowledges support from NASA grants NNX15AH59G and NNX14AG93G and NSF grant AST-1824869.

Facilities: CFHT, CANFAR.

Software: matplotlib (Hunter 2007), scipy (Jones et al. 2001), Mercury (Chambers 2001), SWIFT (Levison \& Duncan 1994), OSSOS Survey Simulator (Lawler et al. 2018a; Petit et al. 2018).

\section{Appendix \\ Future Prospects for TNO Discovery Surveys}

Determining the significance of the detections sunward and outward of the resonances requires that the detections are from a characterized survey with known selection effects. The OSSOS ensemble of surveys provides a powerful tool for testing different models of the outer solar system. These surveys show that there is a statistically significant population found sunward of the resonances. However, this survey has few detections of these near-resonant objects, which results in a large uncertainty in the statistics and population size. Here we determine the effectiveness of a deeper survey in comparing the sunward and outward populations in these models. We use as an example a survey strategy performed by the HSC on the Subaru Telescope with the same layout and field coverage as the OSSOS.

The HSC Subaru Strategic Program (HSC-SSP) included a TNO search, but the survey cadence was not well suited to TNO identification (Chen et al. 2018). However, the detections from this survey confirm the powerful nature of the HSC in a TNO search survey. The HSC-SSP utilized $180 \mathrm{~s}$ exposures in the $g, r, i, z$, and $Y$ bands. In $r$, the optimal band for TNO searches, the limiting magnitude of the survey was $m_{r}=25.0-25.5$ (Chen et al. 2018). For a TNO search, exposures up to $300 \mathrm{~s}$ can be used to maximize depth while keeping trailing loss minimal (for subarcsecond seeing). With an increased exposure time of $300 \mathrm{~s}$, we expect the HSC would deliver a survey limiting magnitude of $m_{r}=25.5-26.0$. Because of the large area, the HSC-SSP was also conducted in a range of sky conditions, including poorer seeing and transparency than the OSSOS discovery survey. A targeted TNO search would require better conditions, and we are

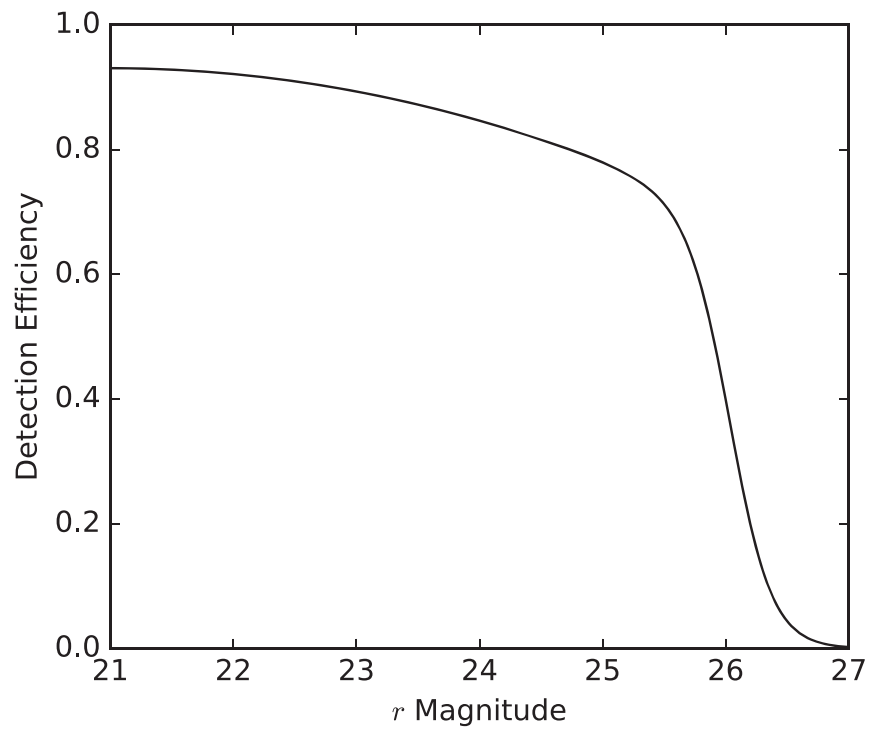

Figure 9. Assumed detection efficiency of the simulated HSC survey blocks. The parameterization is described in Bannister et al. $(2016,2018)$ and modeled after the deepest OSSOS block sensitivity (D-block; $m_{r} \simeq 25.2$ ) but with a fainter limiting magnitude. The survey detection limit is $m_{r}=26$, where the detection efficiency drops to $\sim 40 \%$.

confident that those survey blocks would consistently deliver detections to a limiting magnitude $m_{r}=26.0$ or fainter. Subaru is the only $8 \mathrm{~m}$ class telescope that has a wide-field camera: the HSC has a $90^{\prime}$ diameter field of view. The HSC would also provide a faint limiting magnitude in average observing conditions; OSSOS was able to attain $m_{r} \simeq 25.2$ in the best seeing conditions with the $3.6 \mathrm{~m}$ aperture CFHT. The HSC can provide the optimal system for pushing the frontiers of TNO science.

We use the known characteristics of the HSC to define a simple characterization of a discovery survey on Subaru. To simplify the comparison with current surveys, we use the same field pointings and areas as the OSSOS surveys (described by Bannister et al. 2016, 2018). We expect a similar detection efficiency to OSSOS because of the similar chip gaps and expected moving object detection pipeline method. Here this is parameterized using the same characteristics as the OSSOS D-block efficiency but with a fainter dropoff magnitude $\left(m_{r}=26.05\right)$; see Figure 9. We use a magnitude limit of $m_{r}=26.0$ based on $300 \mathrm{~s}$ exposures on the HSC, as discussed above, and characterize the detection efficiency drop based on the OSSOS efficiency curves (Bannister et al. 2016), as this is closely related to the survey cadence and search method. If the discovery survey is conducted in the same cadence as the OSSOS survey, the same moving object detection pipeline (described in Petit et al. 2004) will provide the best TNO identification tool. This characterization provides a tool for us to test the utility of a deeper HSC survey.

We use the KS16 and PL17 TNO distribution models as sample populations for this theoretical survey. To determine the absolute number of detections, a careful scaling of the model is required to ensure that it can be compared to the actual solar system. For each model, we scaled the number of resonant objects inside the 5:2 and 3:1 and the nearby objects so that each resonant population matches the population estimate found by the Canada-France Ecliptic Plane Survey (CFEPS; 
Table 3

Expected Rejectability Results from a Survey to $m_{r} \leqslant 26$

\begin{tabular}{|c|c|c|c|c|c|c|c|c|}
\hline & \multirow{2}{*}{ Model } & \multirow{2}{*}{$\begin{array}{l}\text { No. High- } q \text { Sim. Detections } \\
\text { Mean } \pm \text { Standard Deviation }\end{array}$} & \multirow{2}{*}{$\begin{array}{c}\text { Number of Particles } \\
H_{r}<10\end{array}$} & \multicolumn{5}{|c|}{$a$ Distribution: \% Not Rejectable $(3 \sigma)$} \\
\hline & & & & KS16 GF & KS16 GS & KS16 SmF & KS16 SmS & PL17 \\
\hline \multirow{4}{*}{$5: 2$} & KS16 GF & $36 \pm 6$ & 53,622 & $100 \%$ & $26 \%$ & $73 \%$ & $16 \%$ & $3 \%$ \\
\hline & $\mathrm{KS} 16 \mathrm{SmF}$ & $30 \pm 6$ & 36,540 & $39 \%$ & $35 \%$ & $94 \%$ & $33 \%$ & $8 \%$ \\
\hline & KS16 SmS & $26 \pm 5$ & 72,216 & $42 \%$ & $91 \%$ & $63 \%$ & $100 \%$ & $32 \%$ \\
\hline & PL17 & $15 \pm 4$ & 67,400 & $98 \%$ & $100 \%$ & $100 \%$ & $99 \%$ & $98 \%$ \\
\hline $3: 1$ & KS16 SmF & $17 \pm 4$ & 45,990 & $84 \%$ & $17 \%$ & $100 \%$ & $83 \%$ & $2 \%$ \\
\hline & KS16 SmS & $16 \pm 4$ & 64,400 & $49 \%$ & $9 \%$ & $31 \%$ & $100 \%$ & $1 \%$ \\
\hline & PL17 & $3 \pm 2$ & 23,100 & $87 \%$ & $87 \%$ & $87 \%$ & $86 \%$ & $87 \%$ \\
\hline
\end{tabular}

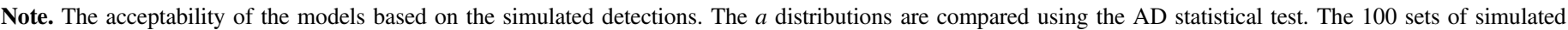

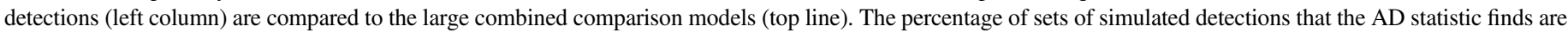

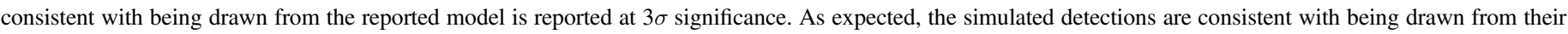
own model. However, the majority of models are statistically inconsistent with each other and could be differentiated by this survey.

Gladman et al. 2012). ${ }^{11}$ The 5:2 resonators are reported to have $12,000_{-8000}^{+15,000}$ members with $H_{r}<8.46$ (here $g$ magnitudes have been transposed to $r$ using $g-r=0.7$, shown to be consistent with the colors of dynamically excited TNOs; Shankman et al. 2016). The 3:1 resonators have $4000_{-3000}^{+9000}$ members in the same size range, according to CFEPS. At pericenter, the faintest detectable objects will have $H_{r}=11.6$ and 11.1 for the 5:2 and 3:1 resonances, respectively. However, the objects of interest are always $q>40$, so we only include objects above that detectability limit, $H_{r}<10$. Assuming the model population $H$-magnitude slope of $\alpha=0.9$ extends to $H_{r}=10$, we expect $68,0005: 2$ objects and 23,000 3:1 resonators with $H_{r}<10$. We duplicated the nonresonant model objects with $q>40$ and within \pm 2 au of the resonance, retaining the $a, e$, and $i$ and randomizing the angular orbital elements in order to produce this large model. For example, Pike et al. (2017) identified 337 5:2 resonators and 77 3:1 resonators in the Brasser \& Morbidelli (2013) simulation, so the numbers of particles near these resonances are scaled by factors of 200 and 300, respectively, to a total of 67,400 and 23,100 particles near the resonances. (See Table 3 for the size of each input near-resonant model.)

We then used the OSSOS Survey Simulator to "observe" these scaled models with our simulated HSC survey and determined the $a$ distribution of detected $q>40$ au objects near the 5:2 and 3:1 resonances. We repeated this process 100 times for each model and resonance to obtain a large sample of high- $q$ a distributions detected by the surveys. A large forwardbiased comparison model was created for each population by combining the simulated detections from all 100 sets. Each of the Survey Simulator runs $(100 \times 5$ models $\times 2$ resonances $)$ was compared to the five large comparison models for that resonance using the AD statistical test (Anderson \& Darling 1954). The significance of the AD statistic was determined using a bootstrapping method. Then, the percentage of the 100 models that were not rejected at $3 \sigma$ significance by the AD statistic was determined. Table 3 summarizes these results: a

\footnotetext{
$\overline{11}$ We note that Volk et al. (2016) provided a more recent estimate of the 5:2 population using CFEPS and the first two blocks of OSSOS, but, due to the way that the population was modeled, the estimate given in that work is a lower limit. A more precise population estimate for the 5:2 based on the entire OSSOS is forthcoming.
}

measurement of whether a deeper survey would be able to discriminate between the different proposed intrinsic models.

The comparison between the different Survey Simulator runs on the models and the larger biased model of each simulation type shows that this deeper survey would successfully provide stronger conclusions about the underlying TNO distribution. There are several main conclusions we draw from the AD statistic results in Table 3. Importantly, the individual Survey Simulator runs are consistent with their biased input model with the statistical significance expected; $\sim 99 \%$ of the models are not rejectable at $3 \sigma$ confidence. When we compare the individual Survey Simulator runs with the biased larger models from other simulations, it is clear that while some models cannot be cleanly differentiated, some are clearly distinct. If the PL17 model is representative of the intrinsic distribution, we will be able to rule out all KS16 model varieties. If the real underlying distribution resembles any of the KS16 models, it appears that we will have less statistical significance in rejecting the PL17 model. However, the significantly fewer detections this model produces are both the reason that these models are not rejected and another criterion we can use to assess rejectability. As a result, a future deeper survey should conclusively determine whether Neptune's migration resembles the KS16 or PL17 models.

Comparing the different KS16 models shows that we may be able to differentiate between some of the specific parameters of Neptune migration. We find that, as expected, models that share components of the same Neptune migration are more similar. For example, the $\mathrm{SmF}$ for both the 5:2 and 3:1 nearresonant particles is similar to the GF and $\mathrm{SmS}$ simulations but may be distinguishable from the GS distribution. Looking at the combination of the 5:2 and 3:1 near-resonant detections will be key to understanding the underlying high- $q$ object distribution.

Here we have assumed a single slope size distribution. Assuming that the high- $q$ TNOs have the same size distribution as other dynamically excited populations, we note that this survey would detect some high- $q$ objects beyond the size distribution transition discovered in several dynamically excited populations (Fraser et al. 2014; Alexandersen et al. 2016; Lawler et al. 2018b). However, the majority of large- $H$ objects in our model are not detected because of their reduced 
brightness, so this effect should not have a significant impact on the results computed here. As a worst-case scenario, we calculate the effect of a size distribution at $H_{r}=8.5$ from $\alpha=0.9$ to 0.5 on the KS16 SmF model, which had the most large- $H$ detections. We find that $60 \%$ of the detections would be retained for this model, resulting in 10 near-resonant detections. When combined with the $\sim 16$ detections of near$5: 2$ objects, these detections would still provide a powerful tool for measuring the underlying near-resonant TNO distribution.

A TNO discovery survey by the HSC would be able to differentiate between the Kaib \& Sheppard (2016) and PL17 models at $2 \sigma-3 \sigma$ significance. Some of the simulations, particularly the KS16 smooth migrations, also produce smaller numbers of near-resonant populations. In addition to future TNO searches, it will be critical to increase dynamical model resolution near the resonances by increasing the number of test particles used. A combination of additional simulations and deeper TNO surveys is required to understand the distribution and history of high- $q$ TNOs.

\section{ORCID iDs}

S. M. Lawler (iD https://orcid.org/0000-0001-5368-386X

R. E. Pike (i) https://orcid.org/0000-0003-4797-5262

M. Alexandersen (i) https://orcid.org/0000-0003-4143-8589

M. T. Bannister (ib https://orcid.org/0000-0003-3257-4490

Y.-T. Chen (陳英同) (1D https://orcid.org/0000-0001-7244-6069

J. J. Kavelaars (iD https://orcid.org/0000-0001-7032-5255

J.-M. Petit (ib https://orcid.org/0000-0003-0407-2266

K. Volk (iD https://orcid.org/0000-0001-8736-236X

\section{References}

Adams, E. R., Gulbis, A. A. S., Elliot, J. L., et al. 2014, AJ, 148, 55 Alexandersen, M., Gladman, B., Kavelaars, J. J., et al. 2016, AJ, 152, 111 Anderson, T. W., \& Darling, D. A. 1954, JASA, 49, 765

Bannister, M. T., Gladman, B. J., Kavelaars, J. J., et al. 2018, ApJS, 236, 18 Bannister, M. T., Kavelaars, J. J., Petit, J.-M., et al. 2016, AJ, 152, 70

Bannister, M. T., Shankman, C., Volk, K., et al. 2017, AJ, 153, 262

Batygin, K., Brown, M. E., \& Fraser, W. C. 2011, ApJ, 738, 13

Brasser, R., \& Morbidelli, A. 2013, Icar, 225, 40

Brasser, R., Morbidelli, A., Gomes, R., Tsiganis, K., \& Levison, H. F. 2009, A\&A, 507, 1053

Chambers, J. E. 2001, Icar, 152, 205

Chen, Y.-T., Lin, H.-W., Alexandersen, M., et al. 2018, PASJ, 70, S38

Dawson, R. I., \& Murray-Clay, R. 2012, ApJ, 750, 43

Duncan, M. J., \& Levison, H. F. 1997, Sci, 276, 1670

Edgeworth, K. E. 1949, MNRAS, 109, 600

Fraser, W. C., Brown, M. E., Morbidelli, A., Parker, A., \& Batygin, K. 2014, ApJ, 782, 100

Gallardo, T., Hugo, G., \& Pais, P. 2012, Icar, 220, 392

Gladman, B. 2005, Sci, 307, 71
Gladman, B., Holman, M., Grav, T., et al. 2002, Icar, 157, 269

Gladman, B., Lawler, S. M., Petit, J.-M., et al. 2012, AJ, 144, 23

Gladman, B., Marsden, B. G., \& Vanlaerhoven, C. 2008, in Nomenclature in the Outer Solar System, ed. M. A. Barucci et al. (Tucson, AZ: Univ. Arizona Press), 43

Gomes, R. S. 2003, Icar, 161, 404

Hahn, J. M., \& Malhotra, R. 2005, AJ, 130, 2392

Holman, M. J., Payne, M. J., Fraser, W., et al. 2018, ApJL, 855, L6

Hunter, J. D. 2007, CSE, 9, 90

Jones, E., Oliphant, T., Peterson, P., et al. 2001, SciPy: Open source scientific tools for Python, https://www.scipy.org/

Jones, R. L., Gladman, B., Petit, J.-M., et al. 2006, Icar, 185, 508

Jones, R. L., Jurić, M., \& Ivezić, Ž 2016, in IAU Symp. 318, Asteroids: New Observations, New Models, ed. S. R. Chesley (Cambridge: Cambridge Univ. Press), 282

Ivezić, Ž, Kahn, S. M., Tyson, J. A., et al. 2019, ApJ, 873, 111

Kaib, N. A., \& Sheppard, S. S. 2016, AJ, 152, 133

Lawler, S. M., \& Gladman, B. 2013, AJ, 146, 6

Lawler, S. M., Kavelaars, J. J., Alexandersen, M., et al. 2018a, FrASS, 5, 14

Lawler, S. M., Shankman, C., Kaib, N., et al. 2017, AJ, 153, 33

Lawler, S. M., Shankman, C., Kavelaars, J. J., et al. 2018b, AJ, 155, 197

Levison, H. F., \& Duncan, M. J. 1994, Icar, 108, 18

Levison, H. F., Morbidelli, A., Vanlaerhoven, C., Gomes, R., \& Tsiganis, K. 2008, Icar, 196, 258

Lykawka, P. S., \& Mukai, T. 2007, Icar, 189, 213

Malhotra, R. 1993, Natur, 365, 819

Malhotra, R., Lan, L., Volk, K., \& Wang, X. 2018, AJ, 156, 55

Morbidelli, A., Brasser, R., Gomes, R., Levison, H. F., \& Tsiganis, K. 2010, AJ, 140, 1391

Nesvorný, D. 2015a, AJ, 150, 73

Nesvorný, D. 2015b, AJ, 150, 68

Nesvorný, D., \& Vokrouhlický, D. 2016, ApJ, 825, 94

Nesvorný, D., Vokrouhlický, D., \& Roig, F. 2016, ApJL, 827, L35

Petit, J., Kavelaars, J. J., Gladman, B., et al. 2011, AJ, 142, 131

Petit, J.-M., Holman, M., Scholl, H., Kavelaars, J., \& Gladman, B. 2004 MNRAS, 347, 471

Petit, J.-M., Kavelaars, J. J., Gladman, B., \& Alexandersen, M. 2018, OSS: OSSOS Survey Simulator, Astrophysics Source Code Library, ascl:1805.014

Petit, J.-M., Kavelaars, J. J., Gladman, B. J., et al. 2017, AJ, 153, 236

Pike, R. E., Kavelaars, J. J., Petit, J. M., et al. 2015, AJ, 149, 202

Pike, R. E., \& Lawler, S. 2017, AJ, 153, 127

Pike, R. E., Lawler, S., Brasser, R., et al. 2017, AJ, 153, 127

Ribeiro de Sousa, R., Gomes, R., Morbidelli, A. r., \& Vieira Neto, E. 2018, arXiv: 1808.02146

Roig, F., \& Nesvorný, D. 2015, AJ, 150, 186

Shankman, C., Gladman, B. J., Kaib, N., Kavelaars, J. J., \& Petit, J. M. 2013, ApJL, 764, L2

Shankman, C., Kavelaars, J., Gladman, B. J., et al. 2016, AJ, 151, 31

Shankman, C., Kavelaars, J. J., Bannister, M. T., et al. 2017a, AJ, 154, 50

Shankman, C., Kavelaars, J. J., Lawler, S. M., Gladman, B. J., \& Bannister, M. T. 2017b, AJ, 153, 63

Shannon, A., \& Dawson, R. I. 2018, MNRAS, 480, 1870

Thommes, E. W., Duncan, M. J., \& Levison, H. F. 1999, Natur, 402, 635

Tsiganis, K., Gomes, R., Morbidelli, A., \& Levison, H. F. 2005, Natur, 435,459

Volk, K., Murray-Clay, R., Gladman, B., et al. 2016, AJ, 152, 23

Volk, K., Murray-Clay, R. A., Gladman, B. J., et al. 2018, AJ, 155, 260

Yu, T. Y. M., Murray-Clay, R., \& Volk, K. 2018, AJ, 156, 33 